\title{
On the Blow-Up of Solutions of a Weakly Dissipative Modified Two-Component Periodic Camassa-Holm System
}

\author{
Yongsheng Mi, ${ }^{1,2}$ Chunlai $\mathrm{Mu}^{1}$ and Weian Tao ${ }^{2}$ \\ ${ }^{1}$ College of Mathematics and Statistics, Chongqing University, Chongqing 400044, China \\ ${ }^{2}$ College of Mathematics and Computer Sciences, Yangtze Normal University, Fuling, \\ Chongqing 408100, China \\ Correspondence should be addressed to Yongsheng Mi, miyongshen@163.com \\ Received 16 May 2012; Revised 24 July 2012; Accepted 30 July 2012 \\ Academic Editor: Ferenc Hartung
}

Copyright (C) 2012 Yongsheng Mi et al. This is an open access article distributed under the Creative Commons Attribution License, which permits unrestricted use, distribution, and reproduction in any medium, provided the original work is properly cited.

We study the Cauchy problem of a weakly dissipative modified two-component periodic CamassaHolm equation. We first establish the local well-posedness result. Then we derive the precise blowup scenario and the blow-up rate for strong solutions to the system. Finally, we present two blowup results for strong solutions to the system.

\section{Introduction}

In this paper, we consider the Cauchy problem of the following weakly dissipative modified two-component Camassa-Holm system:

$$
\begin{gathered}
m_{t}+u m_{x}+2 m u_{x}+\rho \bar{\rho}_{x}+\lambda m=0, \quad t>0, x \in R, \\
\rho_{t}+(\rho u)_{x}+\lambda \rho=0, \quad t>0, x \in R, \\
m(0, x)=m_{0}(x), \quad x \in R, \\
\rho(0, x)=\rho_{0}(x), \quad x \in R, \\
m(t, x+1)=m(t, x), \quad t \geq 0, x \in R, \\
\rho(t, x+1)=\rho(t, x), \quad t \geq 0, x \in R,
\end{gathered}
$$

where $m=\left(1-\partial_{x}^{2}\right) u, \rho=\left(1-\partial_{x}^{2}\right)\left(\bar{\rho}-\bar{\rho}_{0}\right)$, and $\lambda$ is a nonnegative dissipative parameter. 
The Camassa-Holm equation [1] has been recently extended to a two-component integrable system $(\mathrm{CH} 2)$

$$
\begin{gathered}
m_{t}+u m_{x}+2 m u_{x}=\rho \rho_{x}, \quad t>0, x \in R \\
\rho_{t}+(\rho u)_{x}=0, \quad t>0, x \in R
\end{gathered}
$$

with $m=u-u_{x x}$, which is a model for wave motion on shallow water, where $u(t, x)$ describes the horizontal velocity of the fluid, and $\rho(t, x)$ is in connection with the horizontal deviation of the surface from equilibrium, all measured in dimensionless units. Moreover, $u$ and $\rho$ satisfy the boundary conditions: $u \rightarrow 0$ and $\rho \rightarrow 1$ as $|x| \rightarrow \infty$. The system can be identified with the first negative flow of the AKNS hierarchy and possesses the interesting peakon and multikink solutions [2]. Moreover, it is connected with the time-dependent Schrödinger spectral problem [2]. Popowicz [3] observes that the system is related to the bosonic sector of an $N=2$ supersymmetric extension of the classical Camassa-Holm equation. Equation (1.2) with $\rho \equiv 0$ becomes the Camassa-Holm equation, which has global conservative solutions [4] and dissipative solutions [5].

Since the system was derived physically by Constantin and Ivanov [6] in the context of shallow water theory (also by Chen et al. in [2] and Falqui et al. in [7]), many researchers have paid extensive attention to it. In [8], Escher et al. establish the local well-posedness and present the precise blow-up scenarios and several blow-up results of strong solutions to (1.2) on the line. In [6], Constantin and Ivanov investigate the global existence and blow-up phenomena of strong solutions of (1.2) on the line. Later, Guan and Yin [9] obtain a new global existence result for strong solutions to (1.2) and get several blow-up results, which improve the recent results in [6]. Recently, they study the global existence of weak solutions to (1.2) [10]. In [11], Henry studies the infinite propagation speed for (1.2). Gui and Liu [12] establish the local well-posedness for (1.2) in a range of the Besov spaces, they also derive a wave breaking mechanism for strong solutions. Mustafa [13] gives a simple proof of existence for the smooth travelling waves for (1.2). Hu and Yin [14, 15] study the blow-up phenomena and the global existence of (1.2) on the circle.

Recently, the $\mathrm{CH} 2$ system was generalized into the following modified two-component Camassa-Holm (MCH2) system:

$$
\begin{gathered}
m_{t}+u m_{x}+2 m u_{x}=-g \rho \bar{\rho}_{x}, \quad t>0, x \in R \\
\rho_{t}+(\rho u)_{x}=0, \quad t>0, x \in R
\end{gathered}
$$

where $m=\left(1-\partial_{x}^{2}\right) u, \rho=\left(1-\partial_{x}^{2}\right)\left(\bar{\rho}-\bar{\rho}_{0}\right), u$ denotes the velocity field, $\bar{\rho}_{0}$ is taken to be a constant, and $g$ is the downward constant acceleration of gravity in applications to shallow water waves. This $\mathrm{MCH} 2$ system admits peaked solutions in the velocity and average density, we refer this to [16] for details. There, the authors analytically identified the steepening mechanism that allows the singular solutions to emerge from smooth spatially confined initial data. They found that wave breaking in the fluid velocity does not imply singularity in the pointwise density $\rho$ at the point of vertical slope. Some other recent work can be found in [17-25]. We find that the $\mathrm{MCH} 2$ system is expressed in terms of an averaged or filtered density $\bar{\rho}$ in analogy to the relation between momentum and velocity by setting 
$\rho=\left(1-\partial_{x}^{2}\right)\left(\bar{\rho}-\bar{\rho}_{0}\right)$, but it may not be integrable unlike the $\mathrm{CH} 2$ system. The important point here is that $\mathrm{MCH} 2$ has the following conservation law:

$$
\int_{R}\left(u^{2}+u_{x}^{2}+\rho^{2}+\rho_{x}^{2}\right) d x
$$

which play a crucial role in the study of (1.3). Noting that for the $\mathrm{CH} 2$ system, we cannot obtain the conservation of $H^{1}$ norm.

In general, it is quite difficult to avoid energy dissipation mechanisms in a real world. Ghidaglia [26] studies the long time behaviour of solutions to the weakly dissipative KdV equation as a finite-dimensional dynamical system. Recently, $\mathrm{Hu}$ and Yin [27] study the blow-up and blow-up rate of solutions to a weakly dissipative periodic rod equation. In [28, 29], Hu considered global existence and blow-up phenomena for a weakly dissipative two-component Camassa-Holm system on the circle and on the line. However, (1.1) on the circle (periodic case) has not been studied yet. The aim of this paper is to study the blowup phenomena of the strong solutions to (1.1). We find that the behavior of solutions to the weakly dissipative modified two-component periodic Camassa-Holm system (1.1) is similar to that of the modified two-component Camassa- Holm system (1.3), such as the local wellposedness and the blow-up scenario. In addition, we also find that the blow-up rate of (1.1) is not affected by the weakly dissipative term, but the occurrence of blow-up of (1.1) is affected by the dissipative parameter.

This paper is organized as follows: In Section 2, we establish local well-posedness of the Cauchy problem associated with (1.1). In Section 3, we derive precise the blowup scenario of strong solution and the blow-up rate. In Section 4, we discuss the blow-up phenomena of (1.1).

\section{Local Well-Posedness}

In this section, by applying Kato's semigroup theory [30], we can obtain the local wellposedness for the Cauchy problem of (1.1) in $H^{s} \times H^{s}, s>3 / 2$, with with $S=R / Z$ (the circle of unit length).

First, we introduce some notations. All spaces of functions are assumed to be over $S$; for simplicity, we drop $S$ in our notation for function spaces if there is no ambiguity. If $A$ is an unbounded operator, we denote by $D(A)$ the domain of $A$. $[A ; B]$ denotes the commutator of two linear operators $A$ and $B .\|\cdot\|_{X}$ denotes the norm of Banach space $X$. We denote the norm and the inner product of $H^{s} ; s \in R^{+}$, by $\|\cdot\|_{s}$ and $(\cdot, \cdot)_{s}$, respectively.

For convenience, we state here Kato's theorem in the form suitable for our purpose.

Consider the following abstract quasilinear evolution equation:

$$
\frac{d u}{d t}+A(u)=f(u), \quad t>0, u(0)=u_{0}
$$

Let $X$ and $Y$ be Hilbert spaces such that $Y$ is continuously and densely embedded in $X$ and let $Q: Y \rightarrow X$ be a topological isomorphism. $L(Y, X)$ denotes the space of all bounded linear operator from $Y$ to $X$ (and we write $L(X)$, if $X=Y$ ). 
Theorem 2.1 (see [30]). Assume that

(i) $A(y) \in L(Y, X)$ for $y \in X$ with

$$
\|(A(y)-A(z)) w\|_{X} \leq \mu_{1}\|y-z\|_{X}\|w\|_{Y}, \quad y, z, w \in Y,
$$

and $A(y) \in G(X, 1, \beta)$ uniformly on bounded sets in $Y$.

(ii) $Q A(y) Q^{-1}=A(y)+B(y)$, where $B(y) \in L(X)$ is bounded, uniformly on bounded sets in Y. Moreover,

$$
\|(B(y)-B(z)) w\|_{X} \leq \mu_{2}\|y-z\|_{Y}\|w\|_{X}, \quad y, z, \in Y, w \in X
$$

(iii) $f: Y \rightarrow Y$ and extends also to a map from $X$ into $X, f$ is bounded on bounded sets in $Y$ and

$$
\begin{array}{ll}
\|f(y)-f(z)\|_{Y} \leq \mu_{3}\|y-z\|_{Y}, & y, z \in Y, \\
\|f(y)-f(z)\|_{X} \leq \mu_{3}\|y-z\|_{X^{\prime}} & y, z \in Y
\end{array}
$$

where, $\mu_{1}, \mu_{2}$, and $\mu_{3}$ depend only on $\max \left\{\|y\|_{X},\|z\|_{X}\right\}$ and $\mu_{4}$ depends only on $\max \left\{\|y\|_{Y},\|z\|_{Y}\right\}$. If the above conditions (i), (ii), and (iii) hold, given $u_{0} \in Y$, there is a maximal $T>0$ depending only on $\left\|u_{0}\right\|_{Y}$ and a unique solution $u$ to (2.1) such that

$$
u=u\left(\cdot, u_{0}\right) \in C([0, T] ; Y) \cap C^{1}([0, T] ; X) .
$$

Moreover, the map $u_{0} \rightarrow u\left(\cdot, u_{0}\right)$ is continuous from $Y$ to $C([0, T] ; Y) \cap C^{1}([0, T] ; X)$.

We now provide the framework in which we will reformulate system (1.1). With $m=$ $u-u_{x x}, \rho=\gamma-\gamma_{x x}$, and $\gamma=\bar{\rho}-\bar{\rho}_{0}$, we can rewrite (1.1) as follows:

$$
\begin{gathered}
m_{t}+u m_{x}+2 m u_{x}+\rho \gamma_{x}+\lambda m=0, \quad t>0, x \in R, \\
\rho_{t}+(\rho u)_{x}+\lambda \rho=0, \quad t>0, \quad x \in R, \\
m(0, x)=u_{0}(x)-u_{0, x x}(x), \quad x \in R, \\
\rho(0, x)=\gamma_{0}(x)-\gamma_{0, x x}(x), \quad x \in R, \\
m(t, x+1)=m(t, x), \quad t \geq 0, \quad x \in R, \\
\rho(t, x+1)=\rho(t, x), \quad t \geq 0, x \in R .
\end{gathered}
$$

Note that if $p(x):=\cosh (x-[x]-(1 / 2)) / 2 \sinh (1 / 2), x \in R$ is the kernel of $\left(1-\partial_{x}^{2}\right)^{-1}$, where $[x]$ stands for the integer part of $x \in R$, then $\left(1-\partial_{x}^{2}\right)^{-1} f=p * f$ for all $f \in L^{2}(S), p * m=u$, 
and $p * \rho=\gamma$. Here we denote by $*$ the convolution. Using this identity, we can rewrite (2.6) as follows:

$$
\begin{gathered}
u_{t}+u u_{x}=-\partial_{x} p *\left(u^{2}+\frac{1}{2} u_{x}^{2}+\frac{1}{2} \gamma^{2}-\frac{1}{2} \gamma_{x}^{2}\right)-\lambda u, \quad t>0, x \in R, \\
\gamma_{t}+u \gamma_{x}=-p *\left(\left(u_{x} \gamma_{x}\right)_{x}+u_{x} \gamma\right)-\lambda \gamma, \quad t>0, x \in R, \\
u(0, x)=u_{0}(x), \quad x \in R, \\
r(0, x)=\gamma_{0}(x), \quad x \in R, \\
u(t, x+1)=u(t, x), \quad t \geq 0, x \in R, \\
r(t, x+1)=\gamma(t, x), \quad t \geq 0, x \in R,
\end{gathered}
$$

or we can write it in the following equivalent form:

$$
\begin{gathered}
u_{t}+u u_{x}=-\partial_{x}\left(1-\partial_{x}^{2}\right)^{-1}\left(u^{2}+\frac{1}{2} u_{x}^{2}+\frac{1}{2} \gamma^{2}-\frac{1}{2} \gamma_{x}^{2}\right)-\lambda u, \quad t>0, x \in R, \\
\gamma_{t}+u \gamma_{x}=-\partial_{x}\left(1-\partial_{x}^{2}\right)^{-1}\left(u_{x} \gamma_{x}\right)-\left(1-\partial_{x}^{2}\right)^{-1} u_{x} \gamma-\lambda \gamma, \quad t>0, x \in R, \\
u(0, x)=u_{0}(x), \quad x \in R \\
\gamma(0, x)=\gamma_{0}(x), \quad x \in R \\
u(t, x+1)=u(t, x), \quad t \geq 0, x \in R \\
\gamma(t, x+1)=\gamma(t, x), \quad t \geq 0, x \in R
\end{gathered}
$$

Theorem 2.2. Given $z_{0}=z(x, 0)=\left(u_{0}, \gamma_{0}\right) \in H^{s} \times H^{s} s>3 / 2$, then there exist a maximal $T=T\left(z_{0}\right)>0$ and a unique solution $z=(u, \gamma)$ to (1.1) or (2.7) such that

$$
z=z\left(\cdot, z_{0}\right) \in C\left([0, T) ; H^{s} \times H^{s}\right) \cap C^{1}\left([0, T) ; H^{s-1} \times H^{s-1}\right) .
$$

Moreover, the solution depends continuously on the initial data, that is, the mapping $z_{0} \rightarrow z\left(\cdot, z_{0}\right)$ : $H^{s} \times H^{s} \rightarrow C\left([0, T) ; H^{s} \times H^{s}\right) \cap C^{1}\left([0, T) ; H^{s-1} \times H^{s-1}\right)$ is continuous and the maximal time of existence $T>0$ can be chosen to be independent of $s$.

The remainder of this section is devoted to the proof of Theorem 2.2.

Let $z=\left(\begin{array}{l}u \\ \gamma\end{array}\right), A(z)=\left(\begin{array}{cc}u \partial_{x}, & 0 \\ 0, & u \partial_{x}\end{array}\right)$ and

$$
f(z)=\left(\begin{array}{l}
-\partial_{x}\left(1-\partial_{x}^{2}\right)^{-1}\left(u^{2}+\frac{1}{2} u_{x}^{2}+\frac{1}{2} \gamma^{2}-\frac{1}{2} \gamma_{x}^{2}\right)-\lambda u \\
-\partial_{x}\left(1-\partial_{x}^{2}\right)^{-1}\left(u_{x} \gamma_{x}\right)-\left(1-\partial_{x}^{2}\right)^{-1} u_{x} \gamma-\lambda \gamma .
\end{array}\right) .
$$


Set $Y=H^{s} \times H^{s}, X=H^{s-1} \times H^{s-1}, \Lambda=\left(1-\partial_{x}^{2}\right)^{1 / 2}$ and $Q=\left(\begin{array}{cc}\Lambda & 0 \\ 0 & \Lambda\end{array}\right)$. Obviously, $Q$ is an isomorphism of $H^{s} \times H^{s}$ onto $H^{s-1} \times H^{s-1}$. In order to prove Theorem 2.2 by applying Theorem 2.1, we only need to verify $A(z)$ and $f(z)$ which satisfy the conditions (i)-(iii).

We break the argument into several lemmas.

Lemma 2.3. The operator $A(z)=\left(\begin{array}{cc}u \partial_{x}, & 0 \\ 0, & u \partial_{x}\end{array}\right)$, with $z \in H^{s} \times H^{s}, s>3 / 2$, belongs to $G\left(L^{2} \times L^{2}, 1, \beta\right)$.

Lemma 2.4. The operator $A(z)=\left(\begin{array}{cc}u \partial_{x}, & 0 \\ 0, & u \partial_{x}\end{array}\right)$, with $z \in H^{s} \times H^{s}, s>3 / 2$, belongs to $G\left(H^{s-1} \times\right.$ $\left.H^{s-1}, 1, \beta\right)$.

Lemma 2.5. $A(z)=\left(\begin{array}{cc}u \partial_{x}, & 0 \\ 0, & u \partial_{x}\end{array}\right)$, with $z \in H^{s} \times H^{s}, s>3 / 2$. The operator $A(z) \in L\left(H^{s} \times H^{s}, H^{s-1} \times\right.$ $\left.H^{s-1}\right)$. Moreover,

$$
\|(A(y)-A(z)) w\|_{H^{s-1} \times H^{s-1}} \leq \mu_{1}\|y-z\|_{H^{s} \times H^{s}}\|w\|_{H^{s} \times H^{s}}, \quad y, z, w \in \times H^{s} .
$$

Lemma 2.6. The operator $B(z)=[Q, A(z)] Q^{-1}$ with $z \in H^{s} \times H^{s}, s>3 / 2$. Then $B(z) \in L\left(H^{s-1} \times\right.$ $\left.H^{s-1}\right)$ and

$$
\|(B(y)-B(z)) w\|_{H^{s-1} \times H^{s-1}} \leq \mu_{2}\|y-z\|_{H^{s} \times H^{s}}\|w\|_{H^{s-1} \times H^{s-1}},
$$

for $y, z \in H^{s} \times H^{s}$ and $w \in H^{s-1} \times H^{s-1}$. it here.

The proof of the above five lemmas can be done similarly as in [8], therefore we omit

Hence, according to Kato's theorem (Theorem 2.1), in order to prove Theorem 2.2, we only need to verify condition (iii), that is, we need to prove the following lemma.

Lemma 2.7. Let $z \in H^{s} \times H^{s}, s>3 / 2$ and

$$
f(z)=\left(\begin{array}{l}
-\partial_{x}\left(1-\partial_{x}^{2}\right)^{-1}\left(u^{2}+\frac{1}{2} u_{x}^{2}+\frac{1}{2} \gamma^{2}-\frac{1}{2} \gamma_{x}^{2}\right)-\lambda u \\
-\partial_{x}\left(1-\partial_{x}^{2}\right)^{-1}\left(u_{x} \gamma_{x}\right)-\left(1-\partial_{x}^{2}\right)^{-1} u_{x} \gamma-\lambda \gamma
\end{array}\right)
$$

Then $f$ is bounded on bounded sets in $H^{s} \times H^{s}$ and satisfies

(a) $\|f(y)-f(z)\|_{H^{s} \times H^{s}} \leq \mu_{3}\|y-z\|_{H^{s} \times H^{s}}, \quad y, z \in H^{s} \times H^{s}$,

(b) $\|f(y)-f(z)\|_{H^{s-1} \times H^{s-1}} \leq \mu_{4}\|y-z\|_{H^{s-1} \times H^{s-1}}, \quad y, z \in H^{s} \times H^{s}$. 
Journal of Applied Mathematics

Proof. Let $y, z \in H^{s} \times H^{s}, s>3 / 2$. Since $H^{s-1}$ is a Banach algebra, it follows that

$$
\begin{aligned}
\| f(y) & -f(z) \|_{H^{s} \times H^{s}} \\
\leq & \left\|-\partial_{x}\left(1-\partial_{x}^{2}\right)^{-1}\left(\left(y_{1}^{2}-u^{2}\right)+\frac{1}{2}\left(y_{1 x}^{2}-u_{x}^{2}\right)+\frac{1}{2}\left(y_{2}^{2}-\gamma^{2}\right)-\frac{1}{2}\left(y_{2 x}^{2}-\gamma_{x}^{2}\right)\right)-\lambda\left(y_{1}-u\right)\right\|_{H^{s}} \\
& +\left\|-\partial_{x}\left(1-\partial_{x}^{2}\right)^{-1}\left(y_{1 x} y_{2 x}-u_{x} \gamma_{x}\right)-\left(1-\partial_{x}^{2}\right)^{-1}\left(y_{1 x} y_{2}-u_{x} \gamma\right)-\lambda\left(y_{2}-\gamma\right)\right\|_{H^{s}} \\
\leq & \left\|\left(y_{1}-u\right)\left(y_{1}+u\right)\right\|_{H^{s-1}}+\frac{1}{2}\left\|\left(y_{1 x}-u_{x}\right)\left(y_{1 x}+u_{x}\right)\right\|_{H^{s-1}}+\frac{1}{2}\left\|\left(y_{2}-\gamma\right)\left(y_{2}+\gamma\right)\right\|_{H^{s-1}} \\
& +\frac{1}{2}\left\|\left(y_{2 x}-\gamma_{x}\right)\left(y_{2 x}+\gamma_{x}\right)\right\|_{H^{s-1}}+\lambda\left\|y_{1}-u\right\|_{H^{s}}+\left\|u_{x}\left(y_{2 x}-\gamma_{x}\right)\right\|_{H^{s-1}} \\
& +\left\|\left(y_{1 x}-u_{x}\right) y_{2 x}\right\|_{H^{s-1}}+\left\|u_{x}\left(y_{2}-\gamma\right)\right\|_{H^{s-2}}+\left\|\left(y_{1 x}-u_{x}\right) y_{2}\right\|_{H^{s-2}}+\lambda\left\|y_{2}-\gamma\right\| \\
\leq & c\left(\left\|y_{1}-u\right\|_{H^{s-1}}\left\|y_{1}+u\right\|_{H^{s-1}}+\frac{1}{2}\left\|y_{1}-u\right\|_{H^{s}}\left\|y_{1}+u\right\|_{H^{s}}+\frac{1}{2}\left\|y_{2}-\gamma\right\|_{H^{s-1}}\left\|y_{2}+\gamma\right\|_{H^{s-1}}\right) \\
& +\frac{1}{2}\left\|y_{2}-r\right\|_{H^{s}}\left\|y_{2}+\gamma\right\|_{H^{s-1}}+\lambda\left\|y_{1}-u\right\|_{H^{s}}+\|u\|_{H^{s}}\left\|y_{2}-\gamma\right\|_{H^{s}}+\left\|y_{1}-u\right\|_{H^{s}}\left\|y_{2}\right\|_{H^{s}} \\
& +\lambda\left\|y_{2}-\gamma\right\|_{H^{s}}+\|u\|_{H^{s-1}}\left\|y_{2}-\gamma\right\|_{H^{s-2}}+\left\|y_{1}-u\right\|_{H^{s-1}}\left\|y_{2}\right\|_{H^{s-2}} \\
\leq & c\left(\|y\|_{H^{s} \times H^{s}}+\|z\|_{H^{s} \times H^{s}}+\lambda\right)\|y-z\|_{H^{s} \times H^{s}} .
\end{aligned}
$$

This proves (a). Taking $y=0$ in the above inequality, we obtain that $f$ is bounded on bounded set in $H^{s} \times H^{s}$.

Next, we prove (b). Note that $H^{s-1}$ is a Banach algebra. Then, we have

$$
\begin{aligned}
\| f(y) & -f(z) \|_{H^{s-1} \times H^{s-1}} \\
\leq & \left\|-\partial_{x}\left(1-\partial_{x}^{2}\right)^{-1}\left(\left(y_{1}^{2}-u^{2}\right)+\frac{1}{2}\left(y_{1 x}^{2}-u_{x}^{2}\right)+\frac{1}{2}\left(y_{2}^{2}-\gamma^{2}\right)-\frac{1}{2}\left(y_{2 x}^{2}-\gamma_{x}^{2}\right)\right)-\lambda\left(y_{1}-u\right)\right\|_{H^{s-1}} \\
& +\left\|-\partial_{x}\left(1-\partial_{x}^{2}\right)^{-1}\left(y_{1 x} y_{2 x}-u_{x} \gamma_{x}\right)-\left(1-\partial_{x}^{2}\right)^{-1}\left(y_{1 x} y_{2}-u_{x} \gamma\right)-\lambda\left(y_{2}-\gamma\right)\right\|_{H^{s-1}} \\
\leq & \left\|\left(y_{1}-u\right)\left(y_{1}+u\right)\right\|_{H^{s-2}}+\frac{1}{2}\left\|\left(y_{1 x}-u_{x}\right)\left(y_{1 x}+u_{x}\right)\right\|_{H^{s-2}}+\frac{1}{2}\left\|\left(y_{2}-\gamma\right)\left(y_{2}+\gamma\right)\right\|_{H^{s-2}} \\
& +\frac{1}{2}\left\|\left(y_{2 x}-\gamma_{x}\right)\left(y_{2 x}+\gamma_{x}\right)\right\|_{H^{s-2}}+\lambda\left\|y_{1}-u\right\|_{H^{s-1}}+\left\|u_{x}\left(y_{2 x}-\gamma_{x}\right)\right\|_{H^{s-2}} \\
& +\left\|\left(y_{1 x}-u_{x}\right) y_{2 x}\right\|_{H^{s-2}}+\left\|u_{x}\left(y_{2}-\gamma\right)\right\|_{H^{s-3}}+\left\|\left(y_{1 x}-u_{x}\right) y_{2}\right\|_{H^{s-3}}+\lambda\left\|y_{2}-\gamma\right\| \\
\leq & c\left(\left\|y_{1}-u\right\|_{H^{s-2}}\left\|y_{1}+u\right\|_{H^{s-1}}+\frac{1}{2}\left\|y_{1}-u\right\|_{H^{s-1}}\left\|y_{1}+u\right\|_{H^{s-1}}+\frac{1}{2}\left\|y_{2}-\gamma\right\|_{H^{s-2}}\left\|y_{2}+\gamma\right\|_{H^{s-2}}\right)
\end{aligned}
$$




$$
\begin{aligned}
& \quad+\frac{1}{2}\left\|y_{2}-r\right\|_{H^{s-1}}\left\|y_{2}+r\right\|_{H^{s-2}}+\lambda\left\|y_{1}-u\right\|_{H^{s-1}}+\|u\|_{H^{s}}\left\|y_{2}-r\right\|_{H^{s-1}} \\
& \quad+\left\|y_{1}-u\right\|_{H^{s-1}}\left\|y_{2}\right\|_{H^{s-1}}+\lambda\left\|y_{2}-r\right\|_{H^{s-1}}+\|u\|_{H^{s-2}}\left\|y_{2}-r\right\|_{H^{s-3}}+\left\|y_{1}-u\right\|_{H^{s-2}}\left\|y_{2}\right\|_{H^{s-3}} \\
& \leq c\left(\|y\|_{H^{s} \times H^{s-1}}+\|z\|_{H^{s-1} \times H^{s-1}}+\lambda\right)\|y-z\|_{H^{s-1} \times H^{s-1}} .
\end{aligned}
$$

This proves (b) and completes the proof of the Lemma 2.7 .

Proof of Theorem 2.2. Combining Theorem 2.1 and Lemmas 2.3-2.7, we can get the statement of Theorem 2.2.

\section{The Precise Blow-Up Scenario and Blow-Up Rate}

In this section, we present the precise blow-up scenario and the blow-up rate for strong solutions to (2.7).

Lemma 3.1. Let $z_{0}=\left(u_{0}, \gamma_{0}\right) \in H^{s} \times H^{s}, s>3 / 2$, and let $T$ be the maximal existence time of the solution $z=(u, \gamma)$ to $(2.7)$ with the initial data $z_{0}$. Then for all $t \in[0, T)$, we have

$$
\|u(t, \cdot)\|_{H^{1}}^{2}+\|\gamma(t, \cdot)\|_{H^{1}}^{2}=e^{-2 \lambda t}\left(\left\|u_{0}\right\|_{H^{1}}^{2}+\left\|\gamma_{0}\right\|_{H^{1}}^{2}\right) .
$$

Proof. Denote

$$
f(u, \gamma)=u^{2}+\frac{1}{2} u_{x}^{2}+\frac{1}{2} \gamma^{2}-\frac{1}{2} \gamma_{x}^{2}, \quad g=g(u, \gamma)=\left(u_{x} \gamma_{x}\right)_{x}+u_{x} \gamma
$$

In view of the identity $-\partial_{x}^{2} p * f=f-p * f$, we can obtain from (2.7),

$$
u_{t x}=-u_{x}^{2}-u u_{x x}+f-p * f, \quad \gamma_{t x}=-u_{x} \gamma_{x}-u \gamma_{x x}-\partial_{x} p * g
$$

Therefore, an integration by parts yields

$$
\begin{aligned}
\frac{1}{2} \frac{d}{d t}\left(\|u\|_{H^{1}}^{2}+\|\gamma\|_{H^{1}}^{2}\right)= & \int_{R}\left(u u_{t}+u_{x} u_{t x}+\gamma \gamma_{t}+\gamma_{x} \gamma_{t x}\right) d x \\
= & \int_{R} u\left(-u u_{x}-\partial_{x}^{2} p * f-\lambda u\right)+u_{x}\left(-u_{x}^{2}-u u_{x x}+f-p * f-\lambda u_{x}\right) \\
& +\gamma\left(-u \gamma_{x}-p * g-\lambda \gamma\right)+\gamma_{x}\left(-u \gamma_{x}-u \gamma_{x x}-\partial_{x} p * g-\lambda \gamma_{x}\right)
\end{aligned}
$$


Journal of Applied Mathematics

$$
\begin{aligned}
&=\int_{R} {\left[-\frac{1}{2} u_{x}^{3}+u_{x}\left(u^{2}+\frac{1}{2} u_{x}^{2}+\frac{1}{2} \gamma^{2}-\frac{1}{2} \gamma_{x}^{2}\right)-u \gamma \gamma_{x}-\gamma\left(u_{x x} \gamma_{x}+u_{x} \gamma\right)\right.} \\
&\left.\quad-u \gamma_{x} \gamma_{x}^{2}-u \gamma_{x} \gamma_{x x}-\lambda\left(u^{2}+u_{x}^{2}+\gamma^{2}+\gamma_{x}^{2}\right)\right] d x \\
&=-\lambda \int_{R}\left(u^{2}+u_{x}^{2}+\gamma^{2}+\gamma_{x}^{2}\right) d x .
\end{aligned}
$$

Thus, the statement of the conservation law follows.

Lemma 3.2 (see [31]). (i) For every $f \in H^{1}(S)$, we have

$$
\max _{x \in[0,1]} f^{2}(x) \leq \frac{e+1}{2(e-1)}\|f\|_{H^{1}}^{2}
$$

where the constant $(e+1) / 2(e-1)$ is sharp.

(ii) For every $f \in H^{3}(S)$, we have

$$
\max _{x \in[0,1]} f^{2}(x) \leq c\|f\|_{H^{1}}^{2}
$$

with the best possible constant $c$ lying within the range $(1,13 / 12]$. Moreover, the best constant $c$ is $(e+1) / 2(e-1)$.

So, if $z \in H^{3} \times H^{3}$, then by Lemmas 3.1 and 3.2, we have

$$
\begin{aligned}
\|u(t, \cdot)\|_{L^{\infty}}^{2}+\|\gamma(t, \cdot)\|_{L^{\infty}}^{2} & \leq \frac{e+1}{2(e-1)}\|u\|_{H^{1}}^{2}+\frac{e+1}{2(e-1)}\|\gamma\|_{H^{1}}^{2} \\
& =\frac{e+1}{2(e-1)}\left(\left\|u_{0}\right\|_{H^{1}}^{2}+\left\|\gamma_{0}\right\|_{H^{1}}^{2}\right) \\
& =\frac{e+1}{2(e-1)}\left\|z_{0}\right\|_{H^{1} \times H^{1}}^{2}
\end{aligned}
$$

for all $t \in[0, T)$.

Theorem 3.3. Let $z_{0}=(u, \gamma) \in H^{s} \times H^{s}, s>3 / 2$ be given and assume that $T$ is the maximal existence time of the corresponding solution $z=(u, \gamma)$ to (2.7) with initial data $z_{0}$, if there exists $M>0$ such that

$$
\left\|u_{x}(t, \cdot)\right\|_{L^{\infty}}+\left\|\gamma_{x}(t, \cdot)\right\|_{L^{\infty}} \leq M, \quad t \in[0, T)
$$

then the $H^{s} \times H^{s}$ norm of $z(t, \cdot)$ does not blow-up on $[0, T)$. 
The proof of the theorem is similar to the proof of Theorem 3.1 in [20], we omit it here. Consider the following differential equation equation:

$$
\begin{gathered}
\frac{d q(x, t)}{d t}=u(q(x, t), t), \quad t \in[0, T), \\
q(0, t)=x, \quad x \in R,
\end{gathered}
$$

where $u$ denotes the first component of the solution $z$ to (2.7). Applying classical results in the theory of ordinary differential equations, one can obtain the following result on $q$ which is crucial in the proof of blow-up scenario.

Lemma 3.4 (see [8]). Let $u_{0} \in \cap C^{1}\left([0, T) ; H^{s-1}\right), s>3 / 2$, and $T$ be the maximal existence time of the corresponding solution $u(t, x)$ to (3.7). Then (3.7) has a unique solution $q \in C^{1}([0, T) \times R, R)$. Moreover, the map $q(t, \cdot)$ is an increasing diffeomorphism of $R$ with

$$
q_{x}(x, t)=\exp \left(\int_{0}^{t} u_{x}(q(x, s), s) d s\right)>0, \quad q_{x}(x, 0)=1, x \in R, 0 \leq t<T .
$$

The following result is proved only with regard to $r=3$, since we can obtain the same conclusion for the general case $r>3 / 2$ by using Theorem 2.1 and a simple density argument.

We now present a precise blow-up scenario for strong solutions to (2.6).

Theorem 3.5. Let $y_{0}=\left(u_{0}, \gamma_{0}\right) \in H^{s} \times H^{s}, s>3 / 2$, and let $T$ be the maximal existence of the corresponding solution $z=(u, \gamma)$ to (2.7). Then the solution blows up in finite time if and only if

$$
\liminf _{t \rightarrow T, x \in R} u_{x}(t, x)=-\infty \quad \text { or } \quad \limsup _{t \rightarrow T}\left\{\left\|\gamma_{x}(t, \cdot)\right\|_{L^{\infty}}\right\}=+\infty
$$

Proof. Multiplying the first equation in (2.6) by $m=u-u_{x x}$ and integrating by parts, we obtain

$$
\begin{aligned}
\frac{d}{d t} \int_{S} m^{2} d x & =2 \int_{S} m m_{t} d x=2 \int_{S} m\left(-u m_{x}-2 m u_{x}-\rho \gamma_{x}\right) d x-2 \lambda \int_{S} m^{2} d x \\
& =-3 \int_{S} m^{2} u_{x} d x-2 \int_{S} m \rho \gamma_{x} d x-2 \lambda \int_{S} m^{2} d x
\end{aligned}
$$

Repeating the same procedure to the second equation in (2.6) we get

$$
\frac{d}{d t} \int_{S} \rho^{2} d x=-\int_{S} \rho^{2} u_{x}-2 \lambda \int_{S} \rho^{2} d x
$$

A combination of (3.7) and (3.9) yields

$$
\frac{d}{d t} \int_{S}\left(m^{2}+\rho^{2}\right) d x=-3 \int_{S} m^{2} u_{x} d x-2 \int_{S} m \rho r_{x} d x-\int_{S} \rho^{2} u_{x}-2 \lambda \int_{S}\left(m^{2}+\rho^{2}\right) d x
$$


Differentiating the first equation in (2.6) with respect to $x$, multiplying by $m_{x}=u_{x}-u_{x x x}$, then integrating over $S$, we obtain

$$
\begin{aligned}
\frac{d}{d t} \int_{S} m_{x}^{2} d x= & -5 \int_{S} m_{x}^{2} u_{x} d x+2 \int_{S} m^{2} u_{x} d x-2 \int_{S} m_{x} \rho_{x} \gamma_{x} d x \\
& -2 \int_{S} m_{x} \rho \gamma_{x x} d x-2 \lambda \int_{S} m_{x}^{2} d x
\end{aligned}
$$

Similarly,

$$
\frac{d}{d t} \int_{S} \rho_{x}^{2} d x=-3 \int_{S} \rho_{x}^{2} u_{x} d x+\int_{S} \rho^{2} u_{x x x} d x-2 \lambda \int_{S} \rho_{x}^{2} d x
$$

A combination of (3.12)-(3.16) yields

$$
\begin{aligned}
\frac{d}{d t} \int_{S}\left(m^{2}+\rho^{2}+m_{x}^{2}+\rho_{x}^{2}\right) d x \\
=-\int_{S} m^{2} u_{x} d x-5 \int_{S} m_{x}^{2} u_{x} d x-2 \int_{S} m \rho \gamma_{x} d x-2 \int_{S} m_{x} \rho_{x} \gamma_{x} d x-2 \lambda \int_{S}\left(m^{2}+\rho^{2}\right) d x \\
\quad-2 \int_{S} m_{x} \rho \gamma_{x x} d x-\int_{S} \rho^{2} u_{x} d x-3 \int_{S} \rho_{x}^{2} u_{x} d x+\int_{S} \rho^{2} u_{x x x} d x-2 \lambda \int_{S}\left(m_{x}^{2}+\rho_{x}^{2}\right) d x \\
=-\int_{S} m^{2} u_{x} d x-5 \int_{S} m_{x}^{2} u_{x} d x-\int_{S} \rho^{2} u_{x} d x-3 \int_{S} \rho_{x}^{2} u_{x} d x-2 \lambda \int_{S}\left(m^{2}+\rho^{2}\right) d x \\
\quad+\int_{S} \rho^{2} u_{x x x} d x-2 \int_{S} m \rho \gamma_{x} d x-2 \int_{S} m_{x} \rho_{x} \gamma_{x} d x-2 \int_{S} m_{x} \rho \gamma_{x x} d x-2 \lambda \int_{S}\left(m_{x}^{2}+\rho_{x}^{2}\right) d x .
\end{aligned}
$$

Assume that there exists $M_{1}>0$ and $M_{2}>0$ such that $u_{x}(t, x) \geq-M_{1}$ and $\left\|\gamma_{x}(t, \cdot)\right\|_{L^{\infty}} \leq M_{2}$ for all $(t, x) \in[0, T) \times R$, then it follows from Lemma 2.4 that

$$
\|\rho(t, \cdot)\|_{L^{\infty}} \leq e^{M_{1} T}\left\|\rho_{0}(\cdot)\right\|_{L^{\infty}} .
$$

Therefore,

$$
\begin{aligned}
& \frac{d}{d t} \int_{S}\left(m^{2}+\rho^{2}+m_{x}^{2}+\rho_{x}^{2}\right) d x \\
& \quad \leq\left(5 M_{1}\right) \int_{S}\left(m^{2}+\rho^{2}+m_{x}^{2}+\rho_{x}^{2}\right) d x \\
& \quad+\left(M_{2}+e^{M_{1} T}\left\|\rho_{0}(\cdot)\right\|_{L^{\infty}}\right) \int_{S}\left(m^{2}+\rho^{2}+m_{x}^{2}+\rho_{x}^{2}+u_{x x x}^{2}+r_{x x}^{2}\right) d x \\
& \leq\left(5 M_{1}\right) \int_{S}\left(m^{2}+\rho^{2}+m_{x}^{2}+\rho_{x}^{2}\right) d x+2\left(M_{2}+e^{M_{1} T}\left\|\rho_{0}(\cdot)\right\|_{L^{\infty}}\right) \int_{S}\left(m^{2}+\rho^{2}+m_{x}^{2}+\rho_{x}^{2}\right) d x \\
& \leq\left(5 M_{1}+2\left(M_{2}+e^{M_{1} T}\left\|\rho_{0}(\cdot)\right\|_{L^{\infty}}\right)\right) \int_{S}\left(m^{2}+\rho^{2}+m_{x}^{2}+\rho_{x}^{2}\right) d x .
\end{aligned}
$$


The above discussion shows that if there exist $M_{1}>0$ and $M_{2}>0$ such that $u_{x}(t, x) \geq-M_{1}$ and $\left\|\gamma_{x}(t, \cdot)\right\| \leq M_{2}$ for all $(t, x) \in[0, T) \times S$, then there exist two positive constants $K$ and $k$ such that the following estimate holds

$$
\|u(t, \cdot)\|_{H^{s}}^{2}+\|\gamma(t, \cdot)\|_{H^{s}}^{2} \leq K e^{k t}, \quad t \in[0, T) .
$$

This inequality, Sobolev's embedding theorem and Theorem 3.3 guarantee that the solution does not blow-up in finite time.

On the other hand, we see that if

$$
\liminf _{t \rightarrow T, x \in R} u_{x}(t, x)=-\infty \quad \text { or } \limsup _{t \rightarrow T}\left\{\left\|\gamma_{x}(t, \cdot)\right\|_{L^{\infty}}\right\}=+\infty,
$$

then by Sobolev's embedding theorem, the solution will blow-up in finite time. This completes the proof of the theorem.

Lemma 3.6 (see [32]). Let $T>0$ and $v \in C^{1}\left([0, T) ; H^{2}\right)$. Then for every $t \in[0, T)$, there exists at least one point $\xi \in R$ with

$$
\zeta(t):=\inf _{x \in R}\left[v_{x}(t, x)\right]=v_{x}(t, \xi(t)) .
$$

The function $\zeta(t)$ is absolutely continuous on $(0, T)$ with

$$
\frac{d \zeta}{d t}=v_{t x}(t, \xi(t)), \quad \text { a.e., on }(0, T) \text {. }
$$

Theorem 3.7. Let $z_{0}=\left(u_{0}, \gamma_{0}\right) \in H^{s} \times H^{s}, s>3 / 2, z=(u, \gamma)$ be the corresponding solution to (2.7) with initial data $z_{0}$ and satisfies $\left\|\gamma_{x}(t, x)\right\|_{L^{\infty}} \leq M$, for all $(t, x) \in[0, T) \times S, T$ be the maximal existence time of the solution. Then we have

$$
\lim _{t \rightarrow T}\left(\inf _{x \in R} u_{x}(t, x)(T-t)\right)=-2
$$

Proof. Applying Theorems 2.1 and a simple density argument, we only need to show that the above theorem holds for some $s>3 / 2$. Here, we assume $s=3$ to prove the above theorem.

Define now

$$
g(t)=\inf _{x \in s} u_{x}(t, x), \quad t \in[0, T),
$$

and let $\xi \in S$ be a point where this minimum is attained. Clearly, $u_{x x}(t, \xi(t))=0$ since $u(t, \cdot) \in$ $H^{3} \subset C^{2}(S)$. Differentiating the first equation of (2.7) with respect to $x$, in view of $\partial_{x}^{2} p * f=$ $p * f-f$, we have

$$
u_{t x}+u u_{x x}=-\frac{1}{2} u_{x}^{2}+u^{2}+\frac{1}{2} \gamma^{2}-\frac{1}{2} \gamma_{x}^{2}-p *\left(u^{2}+\frac{1}{2} u_{x}^{2}+\frac{1}{2} \gamma^{2}-\frac{1}{2} \gamma_{x}^{2}\right)-\lambda u_{x}
$$


Evaluating (3.26) at $\xi(t)$ and using Lemma 3.6, we obtain

$$
\frac{d}{d t} g(t)+\frac{1}{2} g^{2}(t)+\lambda g(t)=u^{2}(t, \xi(t))+\frac{1}{2} \gamma^{2}(t, \xi(t))-\frac{1}{2} \gamma_{x}^{2}(t, \xi(t))-[p * f](t, \xi(t)),
$$

where $f=u^{2}+(1 / 2) u_{x}^{2}+(1 / 2) \gamma^{2}-(1 / 2) \gamma_{x}^{2}$. By Lemma 3.1 and Young's inequality, we have for all $t \in[0, T)$ that

$$
\begin{aligned}
\|p * f\|_{L^{\infty}} & \leq\|G\|_{L^{\infty}}\left\|u^{2}+\frac{1}{2} u_{x}^{2}+\frac{1}{2} \gamma^{2}-\frac{1}{2} \gamma_{x}^{2}\right\|_{L^{1}} \\
& \leq \frac{\cosh (1 / 2)}{2 \sinh (1 / 2)}\left(\|u\|_{H^{1}}^{2}+\|\gamma\|_{H^{1}}^{2}\right) \\
& =\frac{\cosh (1 / 2)}{2 \sinh (1 / 2)}\|z\|_{H^{1} \times H^{1}}^{2} \leq \frac{\cosh (1 / 2)}{2 \sinh (1 / 2)}\left\|z_{0}\right\|_{H^{1} \times H^{1}}^{2} .
\end{aligned}
$$

This relation together with (3.7) and $\left\|\gamma_{x}(t, x)\right\|_{L^{\infty}} \leq M$ implies that there is a constant $K>0$ such that

$$
\left|g^{\prime}(t)+\frac{1}{2} g(t)+\lambda g(t)\right| \leq K,
$$

where $K$ depends only on $\left\|u_{0}\right\|_{H^{1}}$ and $\left\|\gamma_{0}\right\|_{H^{1}}$. It follows that

$$
-K-\frac{1}{2} \lambda^{2} \leq g^{\prime}(t)+\frac{1}{2}(g(t)+\lambda)^{2} \leq K+\frac{1}{2} \lambda^{2} \quad \text { a.e., on }(0, T)
$$

Choose $\epsilon \in(0,1 / 2)$. Since $\lim \inf _{t \rightarrow T}(y(t)+\lambda)=-\infty$ by Theorem 3.5, there is some $t_{0} \in(0, T)$ with $g\left(t_{0}\right)+\lambda<0$ and $\left(g\left(t_{0}\right)+\lambda\right)^{2}>K+(1 / 2) \lambda^{2} / \epsilon$. Let us first prove that

$$
(g(t)+\lambda)^{2}>\frac{1}{\epsilon}\left(K+\frac{1}{2} \lambda^{2}\right), \quad t \in\left[t_{0}, T\right) .
$$

Since $g$ is locally Lipschitz, there is some $\delta>0$ such that

$$
(g(t)+\lambda)^{2}>\frac{1}{\epsilon}\left(K+\frac{1}{2} \lambda^{2}\right), \quad t \in\left(t_{0}, t_{0}+\delta\right) .
$$

Note that $g$ is locally Lipschitz (it belongs to $W_{\text {loc }}^{1, \infty}(s)$ by Lemma 3.6) and therefore absolutely continuous. Integrating the previous relation on $\left(t_{0}, t_{0}+\delta\right)$ yields that

$$
g\left(t_{0}+\delta\right)+\lambda \leq g\left(t_{0}\right)+\lambda<0
$$

It follows from the above inequality that

$$
\left(g\left(t_{0}+\delta\right)+\lambda\right)^{2} \geq\left(g\left(t_{0}\right)+\lambda\right)^{2}>\frac{1}{\epsilon}\left(K+\frac{1}{2} \lambda^{2}\right) .
$$


The obtained contradiction completes the proof of the relation (3.31). By (3.30)-(3.31), we infer

$$
\frac{1}{2}-\epsilon \leq-\frac{g^{\prime}(t)}{(m+\lambda)^{2}} \leq \frac{1}{2}+\epsilon, \quad \text { a.e. on }(0, T)
$$

For $T \in\left(t_{0}, T\right)$, integrating (3.35) on $(t, T)$ to get

$$
\left(\frac{1}{2}-\epsilon\right)(T-t) \leq-\frac{1}{g(t)+\lambda} \leq\left(\frac{1}{2}+\epsilon\right)(T-t), \quad t \in\left(t_{0}, T\right) .
$$

Since $g(t)+\lambda<0$ on $\left[t_{0}, T\right)$, it follows that

$$
\frac{1}{(1 / 2)+\epsilon} \leq-(g(t)+\lambda)(T-t) \leq \frac{1}{(1 / 2)+\epsilon}, \quad t \in\left(t_{0}, T\right) .
$$

By the arbitrariness of $\epsilon \in(0,1 / 2)$, the statement of the theorem follows.

\section{Blow-Up}

In this section, we discuss the blow-up phenomena of (2.7) and prove that there exist strong solutions to (2.7) which do not exist globally in time.

Theorem 4.1. Let $z_{0}=\left(u_{0}, \gamma_{0}\right) \in H^{s} \times H^{s}, s>3 / 2$ and $T$ be the maximal existence time of the solution $z=(u, \gamma)$ to (2.7) with the initial data $z_{0}$. If there exists some $x_{0} \in S$ such that

$$
u_{0}^{\prime}\left(x_{0}\right)<-\lambda-\sqrt{\lambda^{2}+\left(\frac{e+1}{e-1}+\frac{\cosh (1 / 2)}{2 \sinh (1 / 2)}\right)\left\|z_{0}\right\|_{H^{1} \times H^{1}}^{2}}
$$

then the existence time $T$ is finite and the slope of $u$ tends to negative infinity as $t$ goes to $T$ while $u$ remains uniformly bounded on $[0, T)$.

Proof. As mentioned earlier, here we only need to show that the above theorem holds for $s=3$. Differentiating the first equation of (2.7) with respect to $x$, in view of $\partial_{x}^{2} p * f=p * f-f$, we have

$$
u_{t x}+u u_{x x}=-\frac{1}{2} u_{x}^{2}+u^{2}+\frac{1}{2} \gamma^{2}-\frac{1}{2} \gamma_{x}^{2}-p *\left(u^{2}+\frac{1}{2} u_{x}^{2}+\frac{1}{2} \gamma^{2}-\frac{1}{2} \gamma_{x}^{2}\right)-\lambda u_{x}
$$

Define now

$$
g(t):=\min _{x \in S}\left[u_{x}(t, x)\right], \quad t \in[0, T)
$$

and let $\xi(t) \in S$ be a point where this minimum is attained. It follows that

$$
g(t)=u_{x}(t, \xi(t))
$$


Journal of Applied Mathematics

Clearly $u_{x x}(t, \xi(t))=0$ since $u(t, \cdot) \in H^{3}(S) \subset C^{2}(S)$. Evaluating (4.2) at $\xi(t)$, we obtain

$$
\begin{aligned}
u_{t x}(t, \xi(t))+\frac{1}{2} u_{x}^{2}(t, \xi(t))+\lambda u_{x}(t, \xi(t))= & u^{2}(t, \xi(t))+\frac{1}{2} \gamma^{2}(t, \xi(t))-\frac{1}{2} \gamma_{x}^{2}(t, \xi(t)) \\
& -p *\left(u^{2}+\frac{1}{2} u_{x}^{2}+\frac{1}{2} \gamma^{2}-\frac{1}{2} \gamma_{x}^{2}\right)(t, \xi(t)) \\
\leq & u^{2}(t, \xi(t))+\frac{1}{2} \gamma^{2}(t, \xi(t))+\frac{1}{2} p * r_{x}^{2}(t, \xi(t)) \\
\leq & \frac{e+1}{2(e-1)}\left\|z_{0}\right\|_{H^{1} \times H^{1}}^{2}+\frac{\cosh (1 / 2)}{4 \sinh (1 / 2)}\left\|r_{x}^{2}\right\|_{L^{1}} \\
\leq & \left(\frac{e+1}{2(e-1)}+\frac{\cosh (1 / 2)}{4 \sinh (1 / 2)}\right)\left\|z_{0}\right\|_{H^{1} \times H^{1}}^{2}
\end{aligned}
$$

here, we used Lemma 3.2 and

$$
\left\|p * r_{x}^{2}\right\|_{L^{\infty}} \leq\|p\|_{L^{\infty}}\left\|r_{x}^{2}\right\|_{L^{1}}=\frac{\cosh (1 / 2)}{2 \sinh (1 / 2)}\left\|r_{x}^{2}\right\|_{L^{1}}
$$

Inequality (4.5) and Lemma 3.4 imply

$$
\frac{d}{d t} g(t)+\frac{1}{2} g^{2}(t)+\lambda g(t) \leq\left(\frac{e+1}{2(e-1)}+\frac{\cosh (1 / 2)}{4 \sinh (1 / 2)}\right)\left\|z_{0}\right\|_{H^{1} \times H^{1}}^{2}
$$

that is,

$$
\frac{d}{d t} g(t) \leq-\frac{1}{2} g^{2}(t)-\lambda g(t)+\left(\frac{e+1}{2(e-1)}+\frac{\cosh (1 / 2)}{4 \sinh (1 / 2)}\right)\left\|z_{0}\right\|_{H^{1} \times H^{1}}^{2}
$$

Take

$$
K:=\sqrt{\frac{e+1}{2(e-1)}+\frac{\cosh (1 / 2)}{4 \sinh (1 / 2)}}\left\|z_{0}\right\|_{H^{1} \times H^{1}} .
$$

It then follows that

$$
\begin{aligned}
g^{\prime}(t) & \leq-\frac{1}{2} g^{2}(t)-\lambda g+K^{2} \\
& =-\frac{1}{2}\left(g(t)+\lambda+\sqrt{\lambda^{2}+2 K^{2}}\right)\left(g(t)+\lambda-\sqrt{\lambda^{2}+2 K^{2}}\right) .
\end{aligned}
$$


Note that if $g(0)=u_{0}^{\prime}(\xi(0)) \leq u^{\prime}\left(x_{0}\right) \leq-\lambda-\sqrt{\lambda^{2}+2 K^{2}}$, then $g(t) \leq-\lambda-\sqrt{\lambda^{2}+2 K^{2}}$, for all $t \in[0, T)$. Therefore, we can solve the above inequality to obtain

$$
\frac{g(0)+\lambda+\sqrt{\lambda^{2}+2 K^{2}}}{g(0)+\lambda-\sqrt{\lambda^{2}+2 K^{2}}} e^{\sqrt{\lambda^{2}+2 K^{2}} t}-1 \leq \frac{2 \sqrt{\lambda^{2}+2 K^{2}}}{g(t)+\lambda-\sqrt{\lambda^{2}+2 K^{2}}} \leq 0 .
$$

Due to $0<\left(g(0)+\lambda+\sqrt{\lambda^{2}+2 K^{2}}\right) /\left(g(0)+\lambda-\sqrt{\lambda^{2}+2 K^{2}}\right)<1$, then there exists $T$, and $0<T<$ $\left(1 / \sqrt{\lambda^{2}+2 K^{2}}\right) \ln \left(\left(g(0)+\lambda+\sqrt{\lambda^{2}+2 K^{2}}\right) /\left(g(0)+\lambda-\sqrt{\lambda^{2}+2 K^{2}}\right)\right)$, such that $\lim _{t \rightarrow T} g(t)=-\infty$. This completes the proof of the theorem.

Theorem 4.2. Let $z_{0}=\left(u_{0}, \gamma_{0}\right) \in H^{s} \times H^{s}, s>3 / 2, z=(u, \gamma)$ be the corresponding solution to (2.7) with initial data $z_{0}$ and satisfies $\left\|\gamma_{x}(t, x)\right\|_{L^{\infty}} \leq M$, for all $(t, x) \in[0, T) \times S, T$ be the maximal existence time of the solution. If $z_{0}$ satisfies the following condition:

$$
\int_{S} u_{0 x}^{3} d x<-3 \lambda\left\|z_{0}\right\|_{H^{1} \times H^{1}}^{2}-\sqrt{9 \lambda^{2}\left\|z_{0}\right\|_{H^{1} \times H^{1}}^{4}-2 K^{2}\left\|z_{0}\right\|_{H^{1} \times H^{1}}^{2}}
$$

where $K:=\sqrt{((9(e+1) / 4(e-1))+(3 \cosh (1 / 2) / 4 \sinh (1 / 2)))\left\|z_{0}\right\|_{H^{1} \times H^{1}}^{4}}$. Then the corresponding solution to (2.7) blows up in finite time.

Proof. In view of (4.2), we obtain

$$
\begin{aligned}
\frac{d}{d t} \int_{S} u_{x}^{3} & =3 \int_{S} u_{x}^{2} u_{x t} d x \\
& =3 \int_{S} u_{x}^{2}\left(-u u_{x x}-\frac{1}{2} u_{x}^{2}+u^{2}+\frac{1}{2} \gamma^{2}-\frac{1}{2} \gamma_{x}^{2}-p *\left(u^{2}+\frac{1}{2} u_{x}^{2}+\frac{1}{2} \gamma^{2}-\frac{1}{2} \gamma_{x}^{2}\right)-\lambda u_{x}\right) d x \\
& =3 \int_{S}\left(-u u_{x}^{2} u_{x x}+u_{x}^{2} u^{2}-\frac{1}{2} u_{x}^{4}+\frac{1}{2} u_{x}^{2} \gamma^{2}-\frac{1}{2} u_{x}^{2} r_{x}^{2}-u_{x}^{2} p *\left(u^{2}+\frac{1}{2} u_{x}^{2}+\frac{1}{2} \gamma^{2}\right)\right. \\
& \left.\quad+\frac{1}{2} u_{x}^{2} p * r_{x}^{2}-\lambda u_{x}^{2}\right) d x \\
\leq & 3 \int_{S}\left(-u u_{x}^{2} u_{x x}+u_{x}^{2} u^{2}-\frac{1}{2} u_{x}^{4}+\frac{1}{2} u_{x}^{2} \gamma^{2}+\frac{1}{2} u_{x}^{2} p * r_{x}^{2}-\lambda u_{x}^{3}\right) d x \\
= & -\frac{1}{2} \int_{S} u_{x}^{4} d x+3 \int_{S} u_{x}^{2} u^{2} d x+\frac{3}{2} \int_{S} u_{x} \gamma^{2} d x+\frac{3}{2} \int_{S} u_{x}^{2} p * \gamma_{x}^{2} d x-3 \lambda \int_{S} u_{x}^{3} d x
\end{aligned}
$$


Note that

$$
\begin{gathered}
\left\|p * r_{x}^{2}\right\|_{L^{\infty}} \leq\|G\|_{L^{\infty}}\left\|r_{x}^{2}\right\|_{L^{1}}=\frac{\cosh (1 / 2)}{2 \sinh (1 / 2)}\left\|r_{x}^{2}\right\|_{L^{1^{\prime}}} \\
\int_{S} u_{x}^{2} u^{2} d x \leq\left\|u^{2}\right\|_{L^{\infty}} \int_{S} u_{x}^{2} d x \leq \frac{e+1}{2(e-1)}\left\|z_{0}\right\|_{H^{1} \times H^{1}}^{4}, \\
\int_{S} u_{x}^{2} r^{2} d x \leq\left\|r^{2}\right\|_{L^{\infty}} \int_{S} u_{x}^{2} d x \leq \frac{e+1}{2(e-1)}\left\|z_{0}\right\|_{H^{1} \times H^{1}}^{4}, \\
\int_{S} u_{x}^{2} p * r_{x}^{2} d x \leq \frac{\cosh (1 / 2)}{2 \sinh (1 / 2)}\left\|r_{x}^{2}\right\|_{L^{1}} \int_{S} u_{x}^{2} d x \leq \frac{\cosh (1 / 2)}{2 \sinh (1 / 2)}\left\|z_{0}\right\|_{H^{1} \times H^{1}}^{4} .
\end{gathered}
$$

Thus,

$$
\frac{d}{d t} \int_{S} u_{x}^{3} d x \leq-\frac{1}{2} \int_{S} u_{x}^{4}-3 \lambda \int_{S} u_{x}^{3} d x+\left(\frac{9(e+1)}{4(e-1)}+\frac{3 \cosh (1 / 2)}{4 \sinh (1 / 2)}\right)\left\|z_{0}\right\|_{H^{1} \times H^{1}}^{4}
$$

Using the following inequality:

$$
\left|\int_{S} u_{x}^{3} d x\right| \leq\left(\int_{S} u_{x}^{4} d x\right)^{1 / 2}\left(\int_{S} u_{x}^{2} d x\right)^{1 / 2} \leq\left(\int_{S} u_{x}^{4} d x\right)^{1 / 2}\left\|z_{0}\right\|_{H^{1} \times H^{1}}
$$

and letting

$$
g(t)=\int_{S} u_{x}^{3} d x
$$

we obtain

$$
\frac{d}{d t} g(t) \leq-\frac{1}{2\left\|z_{0}\right\|_{H^{1} \times H^{1}}^{2}} g^{2}(t)-3 \lambda g(t)+\left(\frac{9(e+1)}{4(e-1)}+\frac{3 \cosh (1 / 2)}{4 \sinh (1 / 2)}\right)\left\|z_{0}\right\|_{H^{1} \times H^{1}}^{4} .
$$

Taking

$$
K:=\sqrt{\left(\frac{9(e+1)}{4(e-1)}+\frac{3 \cosh (1 / 2)}{4 \sinh (1 / 2)}\right)\left\|z_{0}\right\|_{H^{1} \times H^{1}}^{4}},
$$

we get

$$
\begin{aligned}
\frac{d}{d t} g(t) \leq & -\frac{1}{2\left\|z_{0}\right\|_{H^{1} \times H^{1}}^{2}}\left(g(t)+3 \lambda\left\|z_{0}\right\|_{H^{1} \times H^{1}}^{2}+\sqrt{9 \lambda^{2}\left\|z_{0}\right\|_{H^{1} \times H^{1}}^{4}+2 K^{2}\left\|z_{0}\right\|_{H^{1} \times H^{1}}^{2}}\right) \\
& \times\left(g(t)+3 \lambda\left\|z_{0}\right\|_{H^{1} \times H^{1}}^{2}-\sqrt{9 \lambda^{2}\left\|z_{0}\right\|_{H^{1} \times H^{1}}^{4}+2 K^{2}\left\|z_{0}\right\|_{H^{1} \times H^{1}}^{2}}\right) .
\end{aligned}
$$


Note that if

$$
g(0)<-3 \lambda\left\|z_{0}\right\|_{H^{1} \times H^{1}}^{2}-\sqrt{9 \lambda^{2}\left\|z_{0}\right\|_{H^{1} \times H^{1}}^{4}+2 K^{2}\left\|z_{0}\right\|_{H^{1} \times H^{1}}^{2}},
$$

then

$$
g(t)<-3 \lambda\left\|z_{0}\right\|_{H^{1} \times H^{1}}^{2}-\sqrt{9 \lambda^{2}\left\|z_{0}\right\|_{H^{1} \times H^{1}}^{4}+2 K^{2}\left\|z_{0}\right\|_{H^{1} \times H^{1}}^{2}},
$$

for all $t \in[0, T)$. From the above inequality, we obtain

$$
\begin{gathered}
\frac{g(0)+3 \lambda\left\|z_{0}\right\|_{H^{1} \times H^{1}}^{2}+\sqrt{9 \lambda^{2}\left\|z_{0}\right\|_{H^{1} \times H^{1}}^{4}+2 K^{2}\left\|z_{0}\right\|_{H^{1} \times H^{1}}^{2}}}{g(0)+3 \lambda\left\|z_{0}\right\|_{H^{1} \times H^{1}}^{2}-\sqrt{9 \lambda^{2}\left\|z_{0}\right\|_{H^{1} \times H^{1}}^{4}+2 K^{2}\left\|z_{0}\right\|_{H^{1} \times H^{1}}^{2}}} e^{\sqrt{9 \lambda^{2}\left\|z_{0}\right\|_{H^{1} \times H^{1}}^{4}+2 K^{2}\left\|z_{0}\right\|_{H^{1} \times H^{1}}^{2}}}-1 \\
\leq \frac{2 \sqrt{9 \lambda^{2}\left\|z_{0}\right\|_{H^{1} \times H^{1}}^{4}+2 K^{2}\left\|z_{0}\right\|_{H^{1} \times H^{1}}^{2}}}{g(t)+3 \lambda\left\|z_{0}\right\|_{H^{1} \times H^{1}}^{2}-\sqrt{9 \lambda^{2}\left\|z_{0}\right\|_{H^{1} \times H^{1}}^{4}+2 K^{2}\left\|z_{0}\right\|_{H^{1} \times H^{1}}^{2}}} \leq 0 .
\end{gathered}
$$

Since $0<\left(g(0)+3 \lambda\left\|z_{0}\right\|_{H^{1} \times H^{1}}^{2}+\sqrt{9 \lambda^{2}\left\|z_{0}\right\|_{H^{1} \times H^{1}}^{4}+2 K^{2}\left\|z_{0}\right\|_{H^{1} \times H^{1}}^{2}}\right) /\left(g(0)+3 \lambda\left\|z_{0}\right\|_{H^{1} \times H^{1}}^{2}-\right.$ $\left.\sqrt{9 \lambda^{2}\left\|z_{0}\right\|_{H^{1} \times H^{1}}^{4}+2 K^{2}\left\|z_{0}\right\|_{H^{1} \times H^{1}}^{2}}\right)<1$ then there exists

$$
\begin{aligned}
0<T \leq & \frac{1}{\sqrt{9 \lambda^{2}\left\|z_{0}\right\|_{H^{1} \times H^{1}}^{4}+2 K^{2}\left\|z_{0}\right\|_{H^{1} \times H^{1}}^{2}}} \\
& \times \ln \left(\frac{g(0)+3 \lambda\left\|z_{0}\right\|_{H^{1} \times H^{1}}^{2}+\sqrt{9 \lambda^{2}\left\|z_{0}\right\|_{H^{1} \times H^{1}}^{4}+2 K^{2}\left\|z_{0}\right\|_{H^{1} \times H^{1}}^{2}}}{g(0)+3 \lambda\left\|z_{0}\right\|_{H^{1} \times H^{1}}^{2}-\sqrt{9 \lambda^{2}\left\|z_{0}\right\|_{H^{1} \times H^{1}}^{4}+2 K^{2}\left\|z_{0}\right\|_{H^{1} \times H^{1}}^{2}}}\right) .
\end{aligned}
$$

such that $\lim _{t \rightarrow T} g(t)=-\infty$. On the other hand,

$$
\left|\int_{S} u_{x}^{3} d x\right| \leq\left\|u_{x}\right\|_{L^{\infty}} \int_{S} u_{x}^{2} \leq\left\|u_{x}\right\|_{L^{\infty}}\|u\| 2_{L^{H^{1}}} \leq\left\|u_{x}\right\|_{L^{\infty}}\left\|z_{0}\right\|_{H^{1} \times H^{1}}^{2}
$$

Applying Theorem 3.5, the solution $z$ blows up in finite time.

\section{Acknowledgments}

This work was partially supported by NSF of China (11071266), Scholarship Award for Excellent Doctoral Student granted by Ministry of Education, and the Educational Science Foundation of Chongqing China (KJ121302). 


\section{References}

[1] R. Camassa and D. D. Holm, "An integrable shallow water equation with peaked solitons," Physical Review Letters, vol. 71, no. 11, pp. 1661-1664, 1993.

[2] M. Chen, S.-Q. Liu, and Y. Zhang, "A two-component generalization of the Camassa-Holm equation and its solutions," Letters in Mathematical Physics, vol. 75, no. 1, pp. 1-15, 2006.

[3] Z. Popowicz, "A 2-component or $N=2$ supersymmetric Camassa-Holm equation," Physics Letters. A, vol. 354, no. 1-2, pp. 110-114, 2006.

[4] H. Holden and X. Raynaud, "Global conservative solutions of the Camassa-Holm equation-a Lagrangian point of view," Communications in Partial Differential Equations, vol. 32, no. 10-12, pp. 1511 $1549,2007$.

[5] H. Holden and X. Raynaud, "Dissipative solutions for the Camassa-Holm equation," Discrete and Continuous Dynamical Systems. Series A, vol. 24, no. 4, pp. 1047-1112, 2009.

[6] A. Constantin and R. I. Ivanov, "On an integrable two-component Camassa-Holm shallow water system," Physics Letters A, vol. 372, no. 48, pp. 7129-7132, 2008.

[7] G. Falqui, "On a Camassa-Holm type equation with two dependent variables," Journal of Physics A, vol. 39, no. 2, pp. 327-342, 2006.

[8] J. Escher, O. Lechtenfeld, and Z. Yin, "Well-posedness and blow-up phenomena for the 2-component Camassa-Holm equation," Discrete and Continuous Dynamical Systems. Series A, vol. 19, no. 3, pp. 493$513,2007$.

[9] C. Guan and Z. Yin, "Global existence and blow-up phenomena for an integrable two-component Camassa-Holm shallow water system," Journal of Differential Equations, vol. 248, no. 8, pp. 2003-2014, 2010.

[10] C. Guan and Z. Yin, "Global weak solutions for a two-component Camassa-Holm shallow water system," Journal of Functional Analysis, vol. 260, no. 4, pp. 1132-1154, 2011.

[11] D. Henry, "Infinite propagation speed for a two component Camassa-Holm equation," Discrete and Continuous Dynamical Systems. Series B, vol. 12, no. 3, pp. 597-606, 2009.

[12] G. Gui and Y. Liu, "On the Cauchy problem for the two-component Camassa-Holm system," Mathematische Zeitschrift, vol. 268, no. 1-2, pp. 45-66, 2011.

[13] O. G. Mustafa, "On smooth traveling waves of an integrable two-component Camassa-Holm shallow water system," Wave Motion, vol. 46, no. 6, pp. 397-402, 2009.

[14] Q. Hu and Z. Yin, "Well-posedness and blow-up phenomena for a periodic two-component CamassaHolm equation," Proceedings of the Royal Society of Edinburgh. Section A, vol. 141, no. 1, pp. 93-107, 2011.

[15] Q. Hu and Z. Yin, "Global existence and blow-up phenomena for a periodic 2-component CamassaHolm equation," Monatshefte für Mathematik, vol. 165, no. 2, pp. 217-235, 2012.

[16] D. D. Holm, L. Ó. Náraigh, and C. Tronci, "Singular solutions of a modified two-component CamassaHolm equation," Physical Review E, vol. 79, no. 1, Article ID 016601, 13 pages, 2009.

[17] G. Lv and M. Wang, "Some remarks for a modified periodic Camassa-Holm system," Discrete and Continuous Dynamical Systems. Series A, vol. 30, no. 4, pp. 1161-1180, 2011.

[18] Y. Fu, Y. Liu, and C. Qu, "Well-posedness and blow-up solution for a modified two-component periodic Camassa-Holm system with peakons," Mathematische Annalen, vol. 348, no. 2, pp. 415-448, 2010.

[19] S. Yu, "Well-posedness and blow-up for a modified two-component Camassa-Holm equation," Applicable Analysis, vol. 91, no. 7, pp. 1321-1337, 2012.

[20] C. Guan, K. H. Karlsen, and Z. Yin, "Well-posedness and blow-up phenomena for a modified two-component Camassa-Holm equation," in Nonlinear Partial Differential Equations and Hyperbolic Wave Phenomena, vol. 526 of Contemporary Mathematics, pp. 199-220, American Mathematical Society, Providence, RI, USA, 2010.

[21] W. Tan and Z. Yin, "Global dissipative solutions of a modified two-component Camassa-Holm shallow water system," Journal of Mathematical Physics, vol. 52, no. 3, Article ID 033507, 24 pages, 2011.

[22] W. Tan and Z. Yin, "Global periodic conservative solutions of a periodic modified two-component Camassa-Holm equation," Journal of Functional Analysis, vol. 261, no. 5, pp. 1204-1226, 2011.

[23] B. He, "New peakon, solitary wave and periodic wave solutions for the modified Camassa-Holm equation," Nonlinear Analysis, vol. 71, no. 12, pp. 6011-6018, 2009.

[24] Z. Guo and M. Zhu, "Wave breaking for a modified two-component Camassa-Holm system," Journal of Differential Equations, vol. 252, no. 3, pp. 2759-2770, 2012. 
[25] L. Jin and Z. Guo, "A note on a modified two-component Camassa-Holm system," Nonlinear Analysis, vol. 13, no. 2, pp. 887-892, 2012.

[26] J.-M. Ghidaglia, "Weakly damped forced Korteweg-de Vries equations behave as a finite-dimensional dynamical system in the long time," Journal of Differential Equations, vol. 74, no. 2, pp. 369-390, 1988.

[27] Q. Hu and Z. Yin, "Blowup and blowup rate of solutions to a weakly dissipative periodic rod equation," Journal of Mathematical Physics, vol. 50, no. 8, Article ID 083503, 16 pages, 2009.

[28] Q. Hu, "Global existence and blow-up phenomena for a weakly dissipative 2-component CamassaHolm system," Applicable Analysis. In press.

[29] Q. Hu, "Global existence and blow-up phenomena for a weakly dissipative periodic 2-component Camassa-Holm system," Journal of Mathematical Physics, vol. 52, no. 10, Article ID 103701, 13 pages, 2011.

[30] T. Kato, "Quasi-linear equations of evolution, with applications to partial differential equations," in Spectral Theory and Differential Equations, vol. 448 of Lecture Notes in Mathematics, pp. 25-70, Springer, Berlin, Germany, 1975.

[31] Z. Yin, "On the blow-up of solutions of the periodic Camassa-Holm equation," Dynamics of Continuous, Discrete E Impulsive Systems. Series A, vol. 12, no. 3-4, pp. 375-381, 2005.

[32] A. Constantin and J. Escher, "Wave breaking for nonlinear nonlocal shallow water equations," Acta Mathematica, vol. 181, no. 2, pp. 229-243, 1998. 


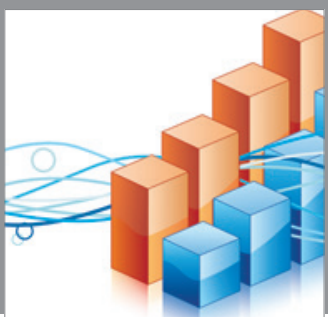

Advances in

Operations Research

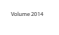

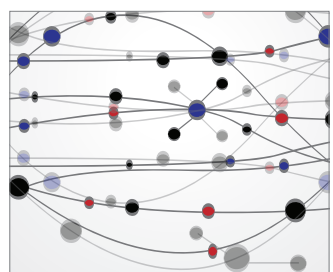

\section{The Scientific} World Journal


International Journal of

Mathematics and

Mathematical

Sciences
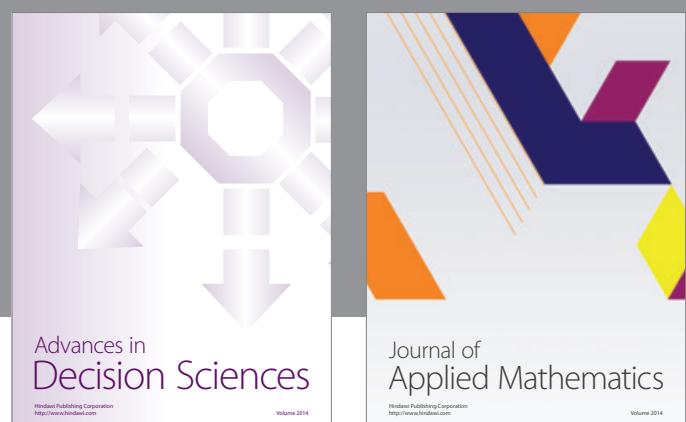

Journal of

Applied Mathematics
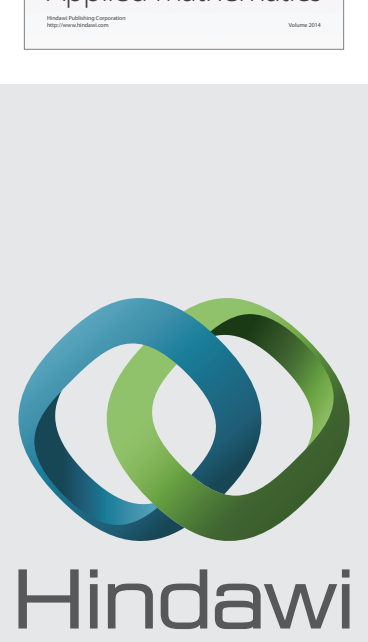

Submit your manuscripts at http://www.hindawi.com


Mathematical Problems in Engineering
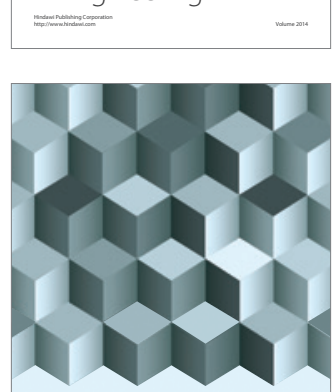

Journal of

Function Spaces
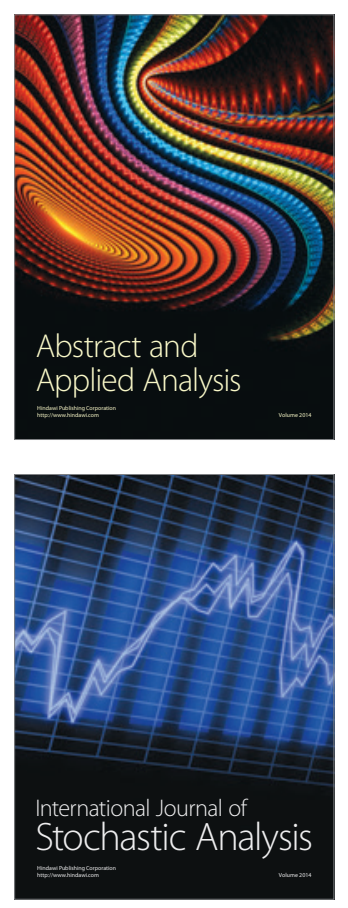

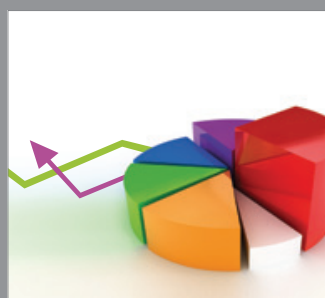

ournal of

Probability and Statistics

Promensencen
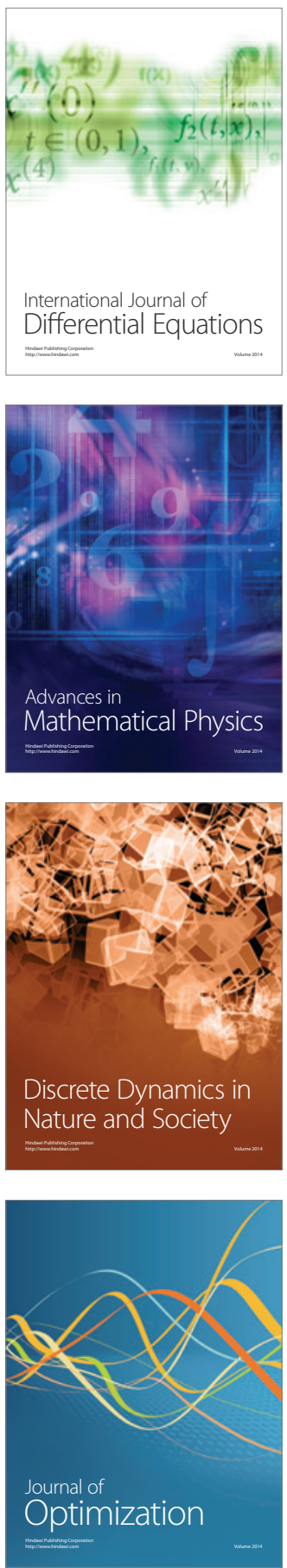